\title{
Acute and chronic toxicities of Bacopa monnieri extract in Sprague-Dawley rats
}

Seewaboon Sireeratawong ${ }^{1,2^{*}}$, Kanjana Jaijoy ${ }^{3}$, Parirat Khonsung ${ }^{1}$, Nirush Lertprasertsuk ${ }^{4}$ and Kornkanok Ingkaninan ${ }^{5}$

\begin{abstract}
Background: Bacopa monnieri is a medicinal plant which has long been used in Ayurvedic medicines to augment brain function and to improve memory. The purpose of our study was to identify and evaluate possible toxic effects of B. monnieri extract in rats by assessing hematological, biochemical, and histopathological parameters.

Methods: Acute oral toxicity of Bacopa monnieri extract was studied in female rats by giving a single orally administered dose at a level of 5,000 mg/kg. The rats were monitored for toxic signs for 14 days. In the chronic toxicity test, groups of both female and male rats were given daily oral doses of $B$. monnieri extract at dose levels of either $30,60,300$ or $1,500 \mathrm{mg} / \mathrm{kg}$ for 270 days. The behavior and health of the animals was then monitored. At the end of the observation period, the body and organ weights of the rats in each group were measured. Blood was collected and necropsy was performed to evaluate their hematology, blood clinical chemistry, and microanatomy.
\end{abstract}

Results: The acute toxicity test found no significant differences between the experimental and the control group rats. In the chronic toxicity test, animal behavior and health of the experimental groups were normal, just as in the control rats. All values of other parameters assessed remained within the normal range.

Conclusion: A single oral administration of B. monnieri extract at the dose of 5,000 mg/kg did not cause any serious undesirable effects. B. monnieri extract at doses of 30,60, 300 and 1,500 mg/kg given for 270 days did not produce any toxicity in rats.

Keywords: Bacopa monnieri, Acute toxicity, Chronic toxicity

\section{Background}

Bacopa monnieri (L.) Wettst, known as Brahmi or water hyssop, (Family Plantaginaceae), is a perennial creeper found in marshy areas throughout Asia where it is used in Ayurvedic medicine for enhancing memory and improving brain function [1]. It has recently been reported that B. monnieri extract has several pharmacological activities, e.g., a neuroprotective effect $[2,3]$, ameliorating cognitive dysfunction [4-6], increasing cerebral blood flow [7], enhancing the activity of antioxidant enzymes and intracellular signaling pathways [8], an antiparkinsonian agent [9], reducing blood pressure [10], hepatoprotection [11], anti-fertility [12], anti-

\footnotetext{
* Correspondence: seewaboon@gmail.com

'Department of Pharmacology, Faculty of Medicine, Chiang Mai University,

Chiang Mai 50200, Thailand

2Division of Pharmacology, Department of Preclinical Science, Faculty of

Medicine, Thammasat University, Pathum Thani 12120, Thailand

Full list of author information is available at the end of the article
}

addiction [13], antioxidant [14, 15], antidepressant [16], anti-stress [17], anti-ulcer [18], anti-cancer [19], and anti-inflammation [20].

Clinical studies have shown that B. monnieri reduces the rate of memory loss of newly acquired information [21], improves memory performance in older persons [22], and enhances cognitive performance in humans [23]. However, B. monnieri was also shown to cause side effects in the gastrointestinal tract, i.e., nausea, increased stool frequency and abdominal cramps [22]. Severe liver toxicity has been reported in a woman after taking several Ayurvedic herbs, including B. monnieri, for nine months; however, after she stopped taking the herbs, her liver function returned to normal [24]. Because of the potential positive ethnobotanical and pharmacological applications of the plant, this study was designed to investigate possible toxic effects of the $B$. monnieri extract in rats. 


\section{Methods}

\section{Plant material and extract}

The B. monnieri was collected in Phetchaburi Province, Thailand, and was identified by Prof. Dr. Wongsatit Chuakul. The voucher specimen (Phrompittayarat 001) is maintained at the PBM Herbarium, Mahidol University, Thailand. An aerial portion of B. monnieri was cut into small pieces, dried $\left(50{ }^{\circ} \mathrm{C}, 12 \mathrm{~h}\right.$.) and then pulverized. The dried powder was percolated twice with $95 \%$ ethanol at the ratio of $1 \mathrm{~g}: 7 \mathrm{ml}$. The percolate was dried in a rotary evaporator under reduced pressure. The yield was $10 \%$ of the fresh weight. The phytochemical study found the extract contained $6.25 \%(\mathrm{w} / \mathrm{w})$ of total saponins, a mixture of $0.87 \%$ of bacoside $\mathrm{A}_{3}, 1.03 \%$ of bacopaside I, $1.82 \%$ of bacopaside II, $0.8 \%$ of bacopaside X, and $1.73 \%$ of bacopasaponin C. $[25,26]$. The extract was kept in a dark bottle in the refrigerator at $5{ }^{\circ} \mathrm{C}$ until used.

\section{Animals}

Male and female Sprague-Dawley rats, 4-5 week-old and weighing 200-250 g, were purchased from the National Laboratory Animal Center (NLAC), Nakorn Pathom, Thailand. All rats were kept in an animal room at a controlled temperature of $25 \pm 1{ }^{\circ} \mathrm{C}$ and with a $12 \mathrm{~h}$ light-dark cycle. A standard rat chow and water ad libitum were provided. The animals were acclimatized for one week before the start of the experiment. The experimental protocols were approved by the Animal Ethics Committee of Faculty of Medicine, Thammasat University (AE011/2552).

\section{Acute toxicity test}

Following OECD Test Guideline 420, in the sighting study female Sprague-Dawley rats received a starting dose of 2,000 mg/kg of extract (Annex 2 - Flow chart of the sighting study), but no toxicity was observed. A main study starting dose of $5,000 \mathrm{mg} / \mathrm{kg}$ was the limit in the acute toxicity study. The female rats were randomly divided into control and treatment groups of five rats each. Rats were fasted overnight prior to dosing on day 1 . Group 1, the control group, received distilled water $(1 \mathrm{~mL} / \mathrm{kg})$ and group 2 , the test group, received $B$. monnieri extract at a dose of $5,000 \mathrm{mg} / \mathrm{kg}$ in a constant volume of $1 \mathrm{~mL} / \mathrm{kg}$ body weight by oral administration.

Observation of toxic signs was recorded systematically for the first $30 \mathrm{~min}$, then periodically during the first $24 \mathrm{~h}$. At the end of the experiment the surviving rats were kept for further 14 days to allow daily observation of clinical signs of toxicity. The body weight of the animals was measured prior to dosing and then weekly after that. On day 15 , the surviving animals were sacrificed using an intraperitoneal overdose of pentobarbital sodium. Their internal organs, including heart, lungs, liver, kidney, spleen, adrenal glands, sex organs and brain, were excised, and weighed and a gross pathological examination was conducted. The organs were then fixed in a $10 \%$ neutral buffered formaldehyde solution for histopathological examination $[27,28]$.

\section{Chronic toxicity test}

The chronic toxicity test was conducted in accordance with WHO and OECD guidelines. The usual dose of $B$. monnieri in food supplements for humans is $300 \mathrm{mg} /$ day or $5 \mathrm{mg} / \mathrm{kg} /$ day (the average body weight of a Thai adult is about $60 \mathrm{~kg}$ ). The dosage of the plant extract used in rats, approximately $30 \mathrm{mg} / \mathrm{kg} /$ day, was calculated based on body surface area [30]. Doses of 30, 60, 300 and $1,500 \mathrm{mg} / \mathrm{kg} /$ day of $B$. monnieri extract were used for the chronic toxicity evaluation. The rats were divided by sex into six groups, ten male and ten female each. All the rats were orally administered either distilled water (control group) or B. monnieri extract once daily for 270 days (experimental group). A satellite group received a dose of $B$. monnieri extract of $1,500 \mathrm{mg} / \mathrm{kg}$ per day for 270 days after which the rats were then reared for an additional 28 days with no $B$. monnieri extract to observe their recovery and to identify any delayed effects of the extract. Animals were weighed and observed daily for toxicological signs, physiological and behavioral changes, as well as mortality. The animals were anesthetized with an intraperitoneal injection of pentobarbital sodium at the end of the experiment. Blood samples were collected from the common carotid artery with an EDTA tube for hematological study and with a non-EDTA tube for biochemical study. Hematological analysis using standard techniques was performed for red blood cell count (RBC), hemoglobin (HB), hematocrit (HCT), mean corpuscular volume $(\mathrm{MCV})$, mean corpuscular hemoglobin $(\mathrm{MCH})$, mean corpuscular hemoglobin concentration (MCHC), platelet count (PLT), white blood cell count (WBC) as well as neutrophil (PMN), lymphocyte (LYMP), monocyte (MONO), eosinophil (EOS), and basophil (BASO) concentrations. Blood samples were biochemically analyzed for levels of glucose (GLU), creatinine (CRE), blood urea nitrogen (BUN), total protein (TP), albumin (ALB), total bilirubin (T-BIL), direct bilirubin (D-BIL), alanine aminotransferase (ALT), aspartate aminotransferase (AST), and alkaline phosphatase (ALP). Finally, the rats were sacrificed and their internal organs were excised, observed, weighed and fixed in $10 \%$ neutral buffered formaldehyde solution for further pathological examination [28, 29].

\section{Statistical analysis}

One-way analysis of variance (ANOVA) and Dunnett's test were performed to determine statistical significance using the SPSS program (version 22.0). In the acute toxicity study, the data were analyzed using Student's $t$-test. Results are expressed as mean \pm standard error of mean (S.E.M.). $P$ values less than 0.05 were considered significant. 


\section{Results}

A large single oral dose of B. monnieri extract $(5,000$ $\mathrm{mg} / \mathrm{kg}$ ) did not cause mortality in rats. Normal behavioral patterns were observed and no changes of eyes, skin, fur, mucous membranes, respiration, circulatory system, autonomic or central nervous systems were found after that dose. The body weight on day 14 of the extract-treated rats had significantly increased compared to that of the control group (Table 1). As shown in Table 2, the average lung weight of treated rats was significantly greater than in the control group, although physical appearance (color and texture) was normal. No significant histopathological change in the internal organs, including the liver and the kidney, were observed (Fig. 1).

In the chronic toxicity study (Fig. 2) a small but significant decrease in the body weight of female rats was observed in the experimental group with some doses of extract compared to the control group (distilled water) measured on the same day. The organ weight of female rats is presented in Table 3. Female B. monnieri extracttreated rats showed the significant greater in the weight of the brain (300 and $1,500 \mathrm{mg} / \mathrm{kg})$, pancreas $(1,500 \mathrm{mg} / \mathrm{kg}$ ) and ovary $(60,300$ and $1,500 \mathrm{mg} / \mathrm{kg})$, while a significant smaller in liver weight $(60 \mathrm{mg} / \mathrm{kg})$ was observed. In male $B$. monnieri extract-treated rats, significant greater in the weight of the lungs $(1,500 \mathrm{mg} / \mathrm{kg})$, kidneys $(30 \mathrm{mg} / \mathrm{kg})$, and epididymes $(60$ and $300 \mathrm{mg} / \mathrm{kg}$ ) were observed. The weight of the heart (satellite group) and liver (60 and $300 \mathrm{mg} / \mathrm{kg}$ ) was significantly lower in male extract-treated groups than in the control group (Table 4).

The effect of B. monnieri extract on hematological parameters is depicted in Tables 5 and 6 . In the female satellite group, the values of RBC, HB, HCT, and MCV were significantly increased. However, $\mathrm{MCH}, \mathrm{MCHC}$ and PLT levels were significantly lower than those of the control group. Moreover, the amounts of WBC and LYMP were significantly increased in female rats treated with B. monnieri extract at doses of 1,500 and $300 \mathrm{mg} / \mathrm{kg}$, respectively. In the male extract-treated groups, the levels of $\mathrm{RBC}(30 \mathrm{mg} / \mathrm{kg}$ ) and $\mathrm{MCHC}$ (satellite group) were significantly decreased, whereas the level of HCT was significantly increased in the satellite group. The amount of WBC, NEU and LYMP was significantly increased in male rats treated with $B$. monnieri extract at doses of 300 and $1,500 \mathrm{mg} / \mathrm{kg}$.

Table 1 Body weight of female rats in acute toxicity test

\begin{tabular}{llll}
\hline & \multicolumn{3}{l}{ Body weight $(\mathrm{g})$} \\
\cline { 2 - 4 } & Day 0 & Day 7 & Day 14 \\
\hline Control & $218.00 \pm 3.74$ & $215.00 \pm 2.24$ & $233.00 \pm 2.00$ \\
B. monnieri extract & $234.00 \pm 4.30$ & $255.00 \pm 6.12$ & $260.00 \pm 7.58^{*}$ \\
$5,000 \mathrm{mg} / \mathrm{kg}$ & & &
\end{tabular}

Values are expressed as mean \pm S.E.M., $n=10$

*Significantly different from control, $p<0.05$
Table 2 Organ weight (g) of female rats in acute toxicity test

\begin{tabular}{lll}
\hline & \multicolumn{2}{l}{ Organ weight $(\mathrm{g})$} \\
\cline { 2 - 3 } & Control & B. monnieri extract $5,000 \mathrm{mg} / \mathrm{kg}$ \\
\hline Brain & $1.79 \pm 0.08$ & $1.73 \pm 0.03$ \\
Lung & $1.27 \pm 0.02$ & $1.44 \pm 0.06^{*}$ \\
Heart & $0.84 \pm 0.05$ & $0.92 \pm 0.03$ \\
Liver & $6.74 \pm 0.29$ & $9.10 \pm 0.61$ \\
Pancreas & $0.62 \pm 0.04$ & $0.86 \pm 0.08$ \\
Spleen & $0.65 \pm 0.04$ & $0.82 \pm 0.04$ \\
Adrenal & $0.04 \pm 0.00$ & $0.04 \pm 0.00$ \\
kidney & $0.83 \pm 0.02$ & $0.86 \pm 0.02$ \\
Ovary & $0.07 \pm 0.00$ & $0.07 \pm 0.00$ \\
Uterus & $0.49 \pm 0.02$ & $0.47 \pm 0.04$
\end{tabular}

Values are expressed as mean \pm S.E.M., $n=10$

*Significantly different from control, $p<0.05$

Clinical blood chemistry parameters were used to clarify the effect of $B$. monnieri extract on the liver (SGPT, SGOT, TP, ALB, ALP, T-BIL and D-BIL), kidney (BUN and CRE), and pancreatic (GLU) function. As shown in Table 7, the level of GLU was significantly decreased in female rats treated with $B$. monnieri extract at doses of 60,300 and $1,500 \mathrm{mg} / \mathrm{kg}$. A small but significant increase of CRE levels and a decrease of TP levels were observed in the female satellite group. In male rats, the level of AST was significantly decreased in groups treated with $B$. monnieri extract at doses of 60,300 and $1,500 \mathrm{mg} / \mathrm{kg}$. In the male satellite group, a small but significant increase of BUN, ALT and ALP levels was found when compared to those of the control group.

No significant histopathological change in the internal organs was found in either the control or the extracttreated rats (Fig. 3).

\section{Discussion}

Toxicity testing is intended to provide information on the safety of an herbal product before further evaluation of its benefits in clinical trials. The appropriate period of administration of the test product to animals is determined by the anticipated period of clinical use in humans [28]. The acute oral toxicity test is used for evaluating any adverse effects appearing within a short time after a single large oral dose of the test substance or after multiple doses given within $24 \mathrm{~h}$. The B. monnieri extract at a dose of $5,000 \mathrm{mg} / \mathrm{kg}$ was selected for acute toxicity testing as an initial dose of $2,000 \mathrm{mg} / \mathrm{kg}$ did not cause any observable signs or symptoms of toxicity. The results showed that $B$. monnieri did not cause death or result in any other signs of toxicity. There was a slight difference in body weight between the control and the treated groups at the beginning of experiment, but it was not statistically significant. However, a small 


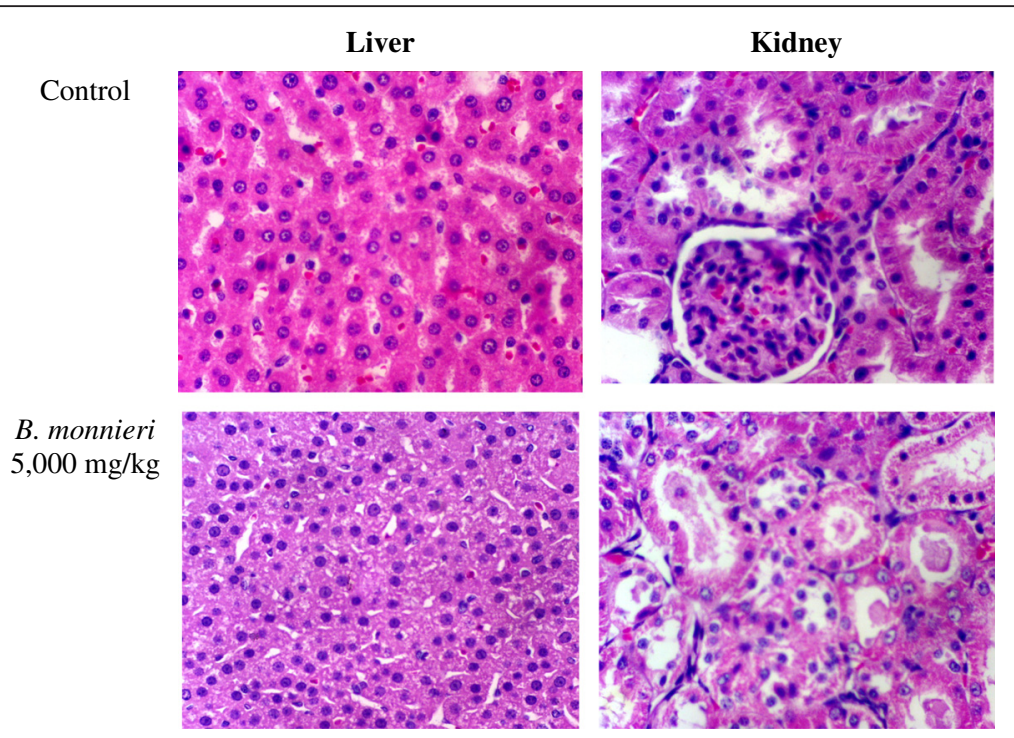

Fig. 1 Histopathology of the liver and kidney of rats in acute toxicity test at 40x magnification (haematoxylin-eosin stain)

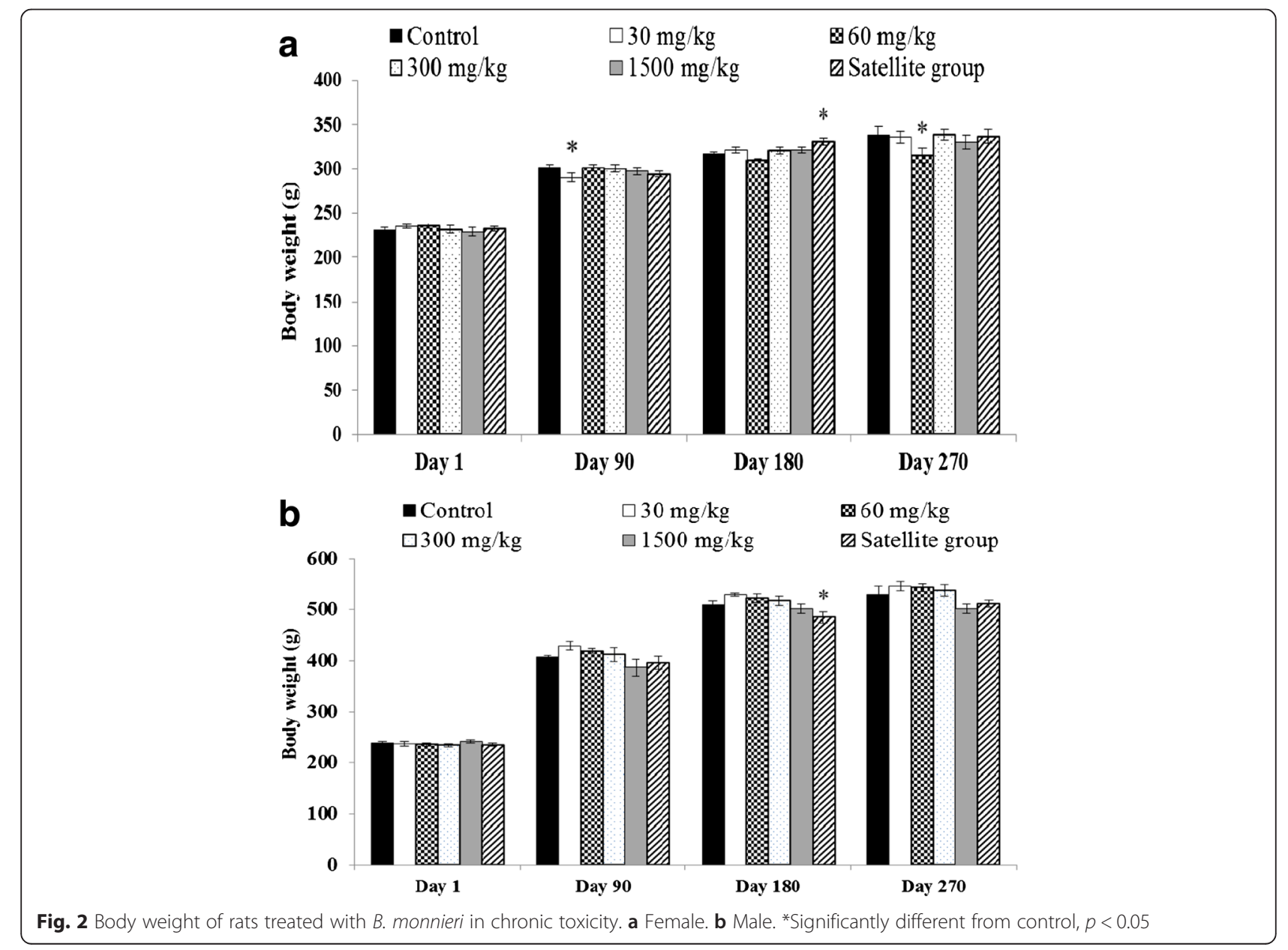


Table 3 Organ weight (g) of female rats in chronic toxicity test

\begin{tabular}{|c|c|c|c|c|c|c|c|c|c|c|c|c|c|c|c|c|c|c|}
\hline \multirow{3}{*}{$\overline{\text { Brain }}$} & \multirow{2}{*}{\multicolumn{3}{|c|}{ Control }} & \multicolumn{15}{|c|}{ B. monnieri extract } \\
\hline & & & & \multicolumn{3}{|c|}{30 mg/kg } & \multicolumn{3}{|c|}{$60 \mathrm{mg} / \mathrm{kg}$} & \multicolumn{3}{|c|}{$300 \mathrm{mg} / \mathrm{kg}$} & \multicolumn{3}{|c|}{$1,500 \mathrm{mg} / \mathrm{kg}$} & \multicolumn{3}{|c|}{ Satellite group } \\
\hline & 1.97 & \pm & 0.02 & 1.94 & \pm & 0.03 & 1.94 & \pm & 0.03 & 2.07 & \pm & $0.04^{*}$ & 2.08 & \pm & $0.02^{*}$ & 2.01 & \pm & 0.03 \\
\hline Lung & 2.16 & \pm & 0.26 & 2.16 & \pm & 0.26 & 2.00 & \pm & 0.14 & 2.14 & \pm & 0.13 & 2.32 & \pm & 0.19 & 2.00 & \pm & 0.13 \\
\hline Heart & 1.27 & \pm & 0.05 & 1.22 & \pm & 0.03 & 1.24 & \pm & 0.04 & 1.38 & \pm & 0.09 & 1.36 & \pm & 0.06 & 1.31 & \pm & 0.05 \\
\hline Liver & 9.90 & \pm & 0.32 & 9.55 & \pm & 0.28 & 8.71 & \pm & $0.30^{*}$ & 9.72 & \pm & 0.23 & 9.33 & \pm & 0.29 & 9.54 & \pm & 0.27 \\
\hline Spleen & 0.83 & \pm & 0.03 & 0.81 & \pm & 0.03 & 0.74 & \pm & 0.03 & 0.83 & \pm & 0.03 & 0.84 & \pm & 0.04 & 0.79 & \pm & 0.04 \\
\hline Pancreas & 1.03 & \pm & 0.09 & 1.09 & \pm & 0.08 & 1.21 & \pm & 0.14 & 1.34 & \pm & 0.13 & 1.42 & \pm & $0.14^{*}$ & 0.98 & \pm & 0.12 \\
\hline Adrenal & 0.04 & \pm & 0.00 & 0.04 & \pm & 0.00 & 0.05 & \pm & 0.00 & 0.04 & \pm & 0.00 & 0.04 & \pm & 0.00 & 0.04 & \pm & 0.00 \\
\hline kidney & 1.27 & \pm & 0.02 & 1.28 & \pm & 0.02 & 1.20 & \pm & 0.03 & 1.22 & \pm & 0.02 & 1.24 & \pm & 0.04 & 1.25 & \pm & 0.03 \\
\hline Ovary & 0.07 & \pm & 0.00 & 0.08 & \pm & 0.00 & 0.09 & \pm & $0.01^{*}$ & 0.09 & \pm & $0.01^{*}$ & 0.09 & \pm & $0.01^{*}$ & 0.07 & \pm & 0.00 \\
\hline Uterus & 1.18 & \pm & 0.14 & 1.36 & \pm & 0.33 & 1.01 & \pm & 0.07 & 0.96 & \pm & 0.09 & 0.86 & \pm & 0.05 & 1.13 & \pm & 0.1 \\
\hline
\end{tabular}

A satellite group was given the $B$. monnieri extract at $1,500 \mathrm{mg} / \mathrm{kg}$ daily over 270 days followed by no treatment for 28 days

Values are expressed as mean \pm S.E.M., $n=10$. *Significantly different from control, $p<0.05$

but significant difference in body weight was observed on day 14. The small increase in body weight of the treated animals can still be accepted as normal, as it can occur due to differences in food consumption.

Neither gross nor histopathological abnormalities were observed in any of the internal organs, including the liver and kidney of B. monnieri-treated rats. This suggests that $B$. monnieri extract is nontoxic at a dose of $5,000 \mathrm{mg} / \mathrm{kg}$. The dose of $B$. monnieri extract used in this study is approximately 1,000 times higher than that generally used in humans $(5 \mathrm{mg} / \mathrm{kg} /$ day $)$. This indicates that $B$. monnieri is quite safe for human use.

A chronic toxicity study normally takes 9 to 12 months as some substances may not display toxicity immediately, but it may appear after repeated exposure. According to WHO guidelines, the period of testing administration in animals should be based on the expected period of clinical use of that substance in humans. The repeated oral administration of the test substance for 9-12 months in animals has been suggested to be comparable to administration for more than 6 months in humans [28].

The objective of chronic toxicity studies is to characterize the profile of the impact of prolonged and repeated exposure to a substance in a mammalian species over a significant portion of the average life span of that species. The study evaluates the impact on target organs including possible accumulation of the substance. The results obtained can then be used as supporting data for designing further clinical trials. The dosage of plant extract for chronic toxicity testing in rats in this study was calculated according to Reagan-Shaw et al. [30]. The B. monnieri extract at doses of $30,60,300$ and $1,500 \mathrm{mg} / \mathrm{kg}$ used in this study are equivalent to 6,12 , 60 , and 300 times of the usual B. monnieri dose in humans, respectively.

Table 4 Organ weight (g) of male rats in chronic toxicity test

\begin{tabular}{|c|c|c|c|c|c|c|c|c|c|c|c|c|c|c|c|c|c|c|}
\hline \multirow[b]{3}{*}{ Brain } & \multirow{2}{*}{\multicolumn{3}{|c|}{ Control }} & \multicolumn{15}{|c|}{ B. monnieri extract } \\
\hline & & & & \multicolumn{3}{|c|}{$30 \mathrm{mg} / \mathrm{kg}$} & \multicolumn{3}{|c|}{$60 \mathrm{mg} / \mathrm{kg}$} & \multicolumn{3}{|c|}{$300 \mathrm{mg} / \mathrm{kg}$} & \multicolumn{3}{|c|}{$1,500 \mathrm{mg} / \mathrm{kg}$} & \multicolumn{3}{|c|}{ Satellite group } \\
\hline & 2.11 & \pm & 0.03 & 2.16 & \pm & 0.03 & 2.16 & \pm & 0.04 & 2.14 & \pm & 0.04 & 2.17 & \pm & 0.04 & 2.11 & \pm & 0.03 \\
\hline Lung & 2.34 & \pm & 0.10 & 2.60 & \pm & 0.13 & 2.58 & \pm & 0.14 & 2.58 & \pm & 0.20 & 3.59 & \pm & $0.21^{*}$ & 2.36 & \pm & 0.13 \\
\hline Heart & 1.84 & \pm & 0.07 & 1.89 & \pm & 0.05 & 1.76 & \pm & 0.03 & 1.79 & \pm & 0.06 & 1.97 & \pm & 0.07 & 1.67 & \pm & $0.06^{*}$ \\
\hline Liver & 15.80 & \pm & 0.44 & 16.80 & \pm & 0.34 & 14.22 & \pm & $0.23^{*}$ & 14.09 & \pm & $0.56^{*}$ & 14.61 & \pm & 0.61 & 14.75 & \pm & 0.37 \\
\hline Pancreas & 0.97 & \pm & 0.06 & 1.05 & \pm & 0.04 & 0.99 & \pm & 0.04 & 1.05 & \pm & 0.04 & 0.98 & \pm & 0.04 & 0.96 & \pm & 0.03 \\
\hline Spleen & 1.27 & \pm & 0.04 & 1.21 & \pm & 0.12 & 1.41 & \pm & 0.14 & 1.08 & \pm & 0.14 & 1.54 & \pm & 0.13 & 0.94 & \pm & 0.09 \\
\hline Adrenal & 0.04 & \pm & 0.00 & 0.04 & \pm & 0.00 & 0.05 & \pm & 0.00 & 0.04 & \pm & 0.00 & 0.04 & \pm & 0.00 & 0.04 & \pm & 0.00 \\
\hline Kidney & 1.86 & \pm & 0.04 & 2.03 & \pm & $0.06^{*}$ & 1.82 & \pm & 0.04 & 1.76 & \pm & 0.04 & 1.76 & \pm & 0.06 & 1.74 & \pm & 0.03 \\
\hline Testis & 2.13 & \pm & 0.03 & 2.11 & \pm & 0.09 & 2.11 & \pm & 0.03 & 2.13 & \pm & 0.03 & 2.05 & \pm & 0.07 & 2.00 & \pm & 0.04 \\
\hline Epididymis & 0.86 & \pm & 0.02 & 0.93 & \pm & 0.03 & 0.99 & \pm & $0.03^{*}$ & 1.04 & \pm & $0.06^{*}$ & 0.93 & \pm & 0.03 & 0.87 & \pm & 0.02 \\
\hline
\end{tabular}

A satellite group was given the $B$. monnieri extract at $1,500 \mathrm{mg} / \mathrm{kg}$ daily over 270 days followed by no treatment for 28 days

Values are expressed as mean \pm S.E.M., $n=10$. *Significantly different from control, $p<0.05$ 
Table 5 Hematological parameters of female rats in chronic toxicity test

\begin{tabular}{|c|c|c|c|c|c|c|c|c|c|c|c|c|c|c|c|c|c|c|}
\hline \multirow{3}{*}{$\begin{array}{l}\text { Hematological } \\
\text { parameter } \\
\mathrm{RBC}\left(\times 10^{6} / \mu \mathrm{l}\right)\end{array}$} & \multirow{2}{*}{\multicolumn{3}{|c|}{ Control }} & \multicolumn{15}{|c|}{ B. monnieri extract } \\
\hline & & & & \multicolumn{3}{|c|}{$30 \mathrm{mg} / \mathrm{kg}$} & \multicolumn{3}{|c|}{$60 \mathrm{mg} / \mathrm{kg}$} & \multicolumn{3}{|c|}{$300 \mathrm{mg} / \mathrm{kg}$} & \multicolumn{3}{|c|}{$1,500 \mathrm{mg} / \mathrm{kg}$} & \multicolumn{3}{|c|}{ Satellite group } \\
\hline & 8.96 & \pm & 0.27 & 9.03 & \pm & 0.37 & 8.80 & \pm & 0.13 & 8.56 & \pm & 0.20 & 8.98 & \pm & 0.18 & 9.92 & \pm & $0.20^{*}$ \\
\hline $\mathrm{HB}(\mathrm{g} / \mathrm{dl})$ & 16.36 & \pm & 0.37 & 16.22 & \pm & 0.30 & 16.24 & \pm & 0.22 & 15.84 & \pm & 0.29 & 16.41 & \pm & 0.27 & 17.50 & \pm & $0.26^{*}$ \\
\hline HCT (\%) & 50.00 & \pm & 1.60 & 50.80 & \pm & 2.12 & 48.80 & \pm & 0.73 & 48.60 & \pm & 1.05 & 50.20 & \pm & 0.89 & 56.60 & \pm & $0.98^{*}$ \\
\hline MCV (fl) & 55.70 & \pm & 0.30 & 56.10 & \pm & 0.43 & 55.40 & \pm & 0.27 & 56.50 & \pm & 0.31 & 55.80 & \pm & 0.59 & 57.00 & \pm & $0.30^{*}$ \\
\hline $\mathrm{MCH}(\mathrm{pg})$ & 18.30 & \pm & 0.22 & 18.09 & \pm & 0.44 & 18.41 & \pm & 0.25 & 18.56 & \pm & 0.27 & 18.30 & \pm & 0.31 & 17.61 & \pm & 0.13 \\
\hline $\mathrm{MCHC}(\mathrm{g} / \mathrm{dl})$ & 32.83 & \pm & 0.45 & 32.18 & \pm & 0.79 & 33.13 & \pm & 0.38 & 32.82 & \pm & 0.37 & 32.75 & \pm & 0.38 & 30.90 & \pm & $0.17^{*}$ \\
\hline $\operatorname{PLT}\left(\times 10^{5} / \mu \mathrm{l}\right)$ & 0.83 & \pm & 0.05 & 0.85 & \pm & 0.05 & 0.77 & \pm & 0.02 & 0.73 & \pm & 0.02 & 0.72 & \pm & 0.09 & 0.68 & \pm & $0.04^{*}$ \\
\hline WBC $\left(\times 10^{3} / \mu \mathrm{l}\right)$ & 5.18 & \pm & 0.34 & 4.96 & \pm & 0.38 & 5.87 & \pm & 0.36 & 6.32 & \pm & 0.61 & 7.03 & \pm & $0.63 *$ & 6.27 & \pm & 0.67 \\
\hline $\operatorname{NEU}\left(\times 10^{3} / \mu \mathrm{l}\right)$ & 1.37 & \pm & 0.16 & 1.28 & \pm & 0.15 & 1.30 & \pm & 0.14 & 1.53 & \pm & 0.42 & 1.41 & \pm & 0.19 & 2.03 & \pm & 0.26 \\
\hline $\operatorname{LYMP}\left(\times 10^{3} / \mu \mathrm{l}\right)$ & 3.18 & \pm & 0.39 & 3.40 & \pm & 0.24 & 4.27 & \pm & 0.28 & 9.59 & \pm & $5.25^{*}$ & 5.31 & \pm & 0.50 & 3.63 & \pm & 0.58 \\
\hline MONO $\left(\times 10^{3} / \mu \mathrm{l}\right)$ & 0.15 & \pm & 0.03 & 0.23 & \pm & 0.06 & 0.21 & \pm & 0.06 & 0.27 & \pm & 0.07 & 0.19 & \pm & 0.03 & 0.23 & \pm & 0.04 \\
\hline $\operatorname{EOS}\left(\times 10^{3} / \mu \mathrm{l}\right)$ & 0.04 & \pm & 0.02 & 0.04 & \pm & 0.03 & 0.09 & \pm & 0.03 & 0.04 & \pm & 0.02 & 0.06 & \pm & 0.02 & 0.03 & \pm & 0.02 \\
\hline $\operatorname{BASO}\left(\times 10^{3} / \mu \mathrm{l}\right)$ & 0.00 & \pm & 0.00 & 0.00 & \pm & 0.00 & 0.00 & \pm & 0.00 & 0.00 & \pm & 0.00 & 0.00 & \pm & 0.00 & 0.00 & \pm & 0.00 \\
\hline
\end{tabular}

A satellite group was given the $B$. monnieri extract at $1,500 \mathrm{mg} / \mathrm{kg}$ daily over 270 days followed by no treatment for 28 days

$R B C$ red blood cell count, $H B$ hemoglobin, $H C T$ hematocrit, $M C V$ mean corpuscular volume, $M C H$ mean corpuscular hemoglobin, $M C H C$ mean corpuscular hemoglobin concentration, PLT platelet count, WBC white blood cell count, NEU neutrophil, LYMP lymphocyte, MONO monocyte, EOS eosinophil, BASO basophil Values are expressed as mean \pm S.E.M., $n=10$. * Significantly different from control, $p<0.05$

In this study, no changes in animal behavior or any toxic signs were observed in the B. monnieri-treated rats. Body weight and internal organ weight were the main parameters used in the assessment of toxicity. Reduction of those two parameters can be used as a sensitivity index of toxicity [31-33]. In this study, the animals were weighed daily throughout the experimental period. Although the weight of some groups of rats decreased or increased slightly, the differences were very small and remained within normal limits. Monitoring the health of the animals during the entire 270 day period found no signs of morbidity or diseases. Rats of both sexes appeared healthy as evidenced by the normal appearance of their general behavior, respiratory patterns, cardiovascular signs, motor activities, reflexes and lack of abnormal changes of skin or fur.

Table 6 Hematological parameters of male rats in chronic toxicity test

\begin{tabular}{|c|c|c|c|c|c|c|c|c|c|c|c|c|c|c|c|c|c|c|}
\hline \multirow{3}{*}{$\begin{array}{l}\text { Hematological } \\
\text { parameter } \\
\text { RBC }\left(\times 10^{6} / \mu \mathrm{l}\right)\end{array}$} & \multirow{2}{*}{\multicolumn{3}{|c|}{ Control }} & \multicolumn{15}{|c|}{ B. monnieri extract } \\
\hline & & & & \multicolumn{3}{|c|}{$30 \mathrm{mg} / \mathrm{kg}$} & \multicolumn{3}{|c|}{$60 \mathrm{mg} / \mathrm{kg}$} & \multicolumn{3}{|c|}{$300 \mathrm{mg} / \mathrm{kg}$} & \multicolumn{3}{|c|}{$1,500 \mathrm{mg} / \mathrm{kg}$} & \multicolumn{3}{|c|}{ Satellite group } \\
\hline & 9.76 & \pm & 0.18 & 8.53 & \pm & $0.89^{*}$ & 10.03 & \pm & 0.30 & 9.53 & \pm & 0.30 & 9.60 & \pm & 0.21 & 10.73 & \pm & 0.20 \\
\hline $\mathrm{HB}(\mathrm{g} / \mathrm{dl})$ & 16.99 & \pm & 0.23 & 16.73 & \pm & 0.50 & 16.92 & \pm & 0.43 & 16.73 & \pm & 0.51 & 16.77 & \pm & 0.41 & 17.82 & \pm & 0.28 \\
\hline HCT (\%) & 52.00 & \pm & 0.91 & 51.00 & \pm & 1.86 & 52.90 & \pm & 1.55 & 50.60 & \pm & 1.58 & 50.50 & \pm & 1.30 & 57.50 & \pm & $0.98^{*}$ \\
\hline $\mathrm{MCV}(\mathrm{fl})$ & 53.00 & \pm & 0.21 & 53.40 & \pm & 0.31 & 52.60 & \pm & 0.27 & 53.00 & \pm & 0.30 & 52.50 & \pm & 0.27 & 53.50 & \pm & 0.31 \\
\hline $\mathrm{MCH}(\mathrm{pg})$ & 17.38 & \pm & 0.17 & 17.54 & \pm & 0.09 & 16.85 & \pm & 0.15 & 17.54 & \pm & 0.11 & 16.41 & \pm & 1.03 & 16.56 & \pm & 0.12 \\
\hline $\mathrm{MCHC}(\mathrm{g} / \mathrm{dl})$ & 32.80 & \pm & 0.28 & 32.91 & \pm & 0.26 & 32.07 & \pm & 0.25 & 33.16 & \pm & 0.19 & 33.18 & \pm & 0.22 & 30.92 & \pm & $0.12^{*}$ \\
\hline $\operatorname{PLT}\left(\times 10^{5} / \mu \mathrm{l}\right)$ & 0.95 & \pm & 0.03 & 0.86 & \pm & 0.03 & 0.97 & \pm & 0.03 & 0.93 & \pm & 0.04 & 1.85 & \pm & 0.89 & 0.89 & \pm & 0.04 \\
\hline WBC $\left(\times 10^{3} / \mu \mathrm{l}\right)$ & 7.70 & \pm & 0.42 & 7.23 & \pm & 0.69 & 9.52 & \pm & 0.40 & 11.62 & \pm & $1.44^{*}$ & 10.01 & \pm & $0.72^{*}$ & 7.79 & \pm & 0.60 \\
\hline $\operatorname{NEU}\left(\times 10^{3} / \mu \mathrm{l}\right)$ & 1.37 & \pm & 0.16 & 1.80 & \pm & 0.23 & 2.12 & \pm & 0.22 & 2.89 & \pm & $1.19^{*}$ & 2.18 & \pm & 0.26 & 2.10 & \pm & 0.26 \\
\hline $\operatorname{LYMP}\left(\times 10^{3} / \mu \mathrm{l}\right)$ & 5.94 & \pm & 0.28 & 5.14 & \pm & 0.52 & 6.90 & \pm & 0.29 & 8.08 & \pm & $0.50^{*}$ & 7.31 & \pm & $0.50^{*}$ & 5.31 & \pm & 0.55 \\
\hline MONO $\left(\times 10^{3} / \mu \mathrm{l}\right)$ & 0.27 & \pm & 0.06 & 0.21 & \pm & 0.07 & 0.30 & \pm & 0.07 & 0.49 & \pm & 0.17 & 0.45 & \pm & 0.12 & 0.35 & \pm & 0.06 \\
\hline $\operatorname{EOS}\left(\times 10^{3} / \mu \mathrm{l}\right)$ & 0.11 & \pm & 0.05 & 0.08 & \pm & 0.03 & 0.20 & \pm & 0.08 & 0.06 & \pm & 0.05 & 0.07 & \pm & 0.03 & 0.02 & \pm & 0.02 \\
\hline BASO $\left(\times 10^{3} / \mu \mathrm{l}\right)$ & 0.00 & \pm & 0.00 & 0.00 & \pm & 0.00 & 0.00 & \pm & 0.00 & 0.00 & \pm & 0.00 & 0.00 & \pm & 0.00 & 0.00 & \pm & 0.00 \\
\hline
\end{tabular}

A satellite group was given the $B$. monnieri extract at $1,500 \mathrm{mg} / \mathrm{kg}$ daily over 270 days followed by no treatment for 28 days

$R B C$ red blood cell count, $H B$ hemoglobin, $H C T$ hematocrit, $M C V$ mean corpuscular volume, $M C H$ mean corpuscular hemoglobin, $M C H C$ mean corpuscular hemoglobin concentration, PLT platelet count, WBC white blood cell count, NEU neutrophil, LYMP lymphocyte, MONO monocyte, EOS eosinophil, BASO basophil Values are expressed as mean \pm S.E.M., $n=10$. *Significantly different from control, $p<0.05$ 
Table 7 Biochemical parameters of female and male rats in chronic toxicity test

\begin{tabular}{|c|c|c|c|c|c|c|c|c|c|c|c|c|c|c|c|c|c|c|}
\hline \multirow{3}{*}{$\begin{array}{l}\text { Biochemical } \\
\text { parameter }\end{array}$} & \multirow[t]{2}{*}{ Control } & & & \multicolumn{15}{|c|}{ B. monnieri extract } \\
\hline & & & & \multicolumn{3}{|c|}{$30 \mathrm{mg} / \mathrm{kg}$} & \multicolumn{3}{|c|}{$60 \mathrm{mg} / \mathrm{kg}$} & \multicolumn{3}{|c|}{300 mg/kg } & \multicolumn{3}{|c|}{$1,500 \mathrm{mg} / \mathrm{kg}$} & \multicolumn{3}{|c|}{ Satellite group } \\
\hline & & & & & & & & & & & & & & & & & & \\
\hline GLU (mg/dl) & 177.30 & \pm & 5.66 & 159.90 & \pm & 7.50 & 147.50 & \pm & $6.55^{*}$ & 134.10 & \pm & $8.50^{*}$ & 132.20 & \pm & $7.96^{*}$ & 170.3 & \pm & 8.85 \\
\hline BUN (mg/dl) & 16.12 & \pm & 1.99 & 16.54 & \pm & 0.41 & 15.76 & \pm & 1.23 & 16.47 & \pm & 0.44 & 27.50 & \pm & 11.31 & 21.32 & \pm & 0.53 \\
\hline CRE (mg/dl) & 0.56 & \pm & 0.02 & 0.57 & \pm & 0.01 & 0.55 & \pm & 0.01 & 0.58 & \pm & 0.01 & 0.60 & \pm & 0.02 & 0.61 & \pm & $0.02^{*}$ \\
\hline $\mathrm{TP}(\mathrm{g} / \mathrm{dl})$ & 6.29 & \pm & 0.08 & 6.20 & \pm & 0.08 & 6.15 & \pm & 0.09 & 6.22 & \pm & 0.12 & 6.16 & \pm & 0.10 & 5.86 & \pm & $0.03^{*}$ \\
\hline ALB (g/dl) & 3.20 & \pm & 0.03 & 3.27 & \pm & 0.03 & 3.27 & \pm & 0.03 & 3.20 & \pm & 0.05 & 3.12 & \pm & 0.04 & 3.18 & \pm & 0.05 \\
\hline T-BIL (mg/dl) & 0.10 & \pm & 0.01 & 0.10 & \pm & 0.01 & 0.11 & \pm & 0.01 & 81.18 & \pm & 81.09 & 0.10 & \pm & 0.00 & 0.25 & \pm & 0.02 \\
\hline D-BIL (mg/dl) & 0.18 & \pm & 0.02 & 0.17 & \pm & 0.02 & 0.23 & \pm & 0.03 & 0.28 & \pm & 0.08 & 0.22 & \pm & 0.03 & 0.11 & \pm & 0.01 \\
\hline AST $(U / L)$ & 195.60 & \pm & 26.12 & 220.20 & \pm & 22.37 & 162.70 & \pm & 9.09 & 213.40 & \pm & 21.86 & 178.20 & \pm & 21.36 & 204.80 & \pm & 21.98 \\
\hline $\operatorname{ALT}(U / L)$ & 57.60 & \pm & 9.00 & 77.70 & \pm & 10.96 & 49.70 & \pm & 5.35 & 73.40 & \pm & 9.83 & 61.10 & \pm & 9.88 & 63.20 & \pm & 7.79 \\
\hline $\mathrm{ALP}(\mathrm{U} / \mathrm{L})$ & 43.20 & \pm & 2.92 & 40.60 & \pm & 3.03 & 41.90 & \pm & 3.22 & 48.00 & \pm & 7.90 & 48.10 & \pm & 6.03 & 43.10 & \pm & 3.94 \\
\hline \multicolumn{19}{|l|}{ Male rats } \\
\hline $\mathrm{GLU}(\mathrm{mg} / \mathrm{dl})$ & 209.10 & \pm & 8.67 & 209.40 & \pm & 15.59 & 181.40 & \pm & 18.86 & 219.60 & \pm & 14.18 & 196.40 & \pm & 9.49 & 174.20 & \pm & 8.07 \\
\hline BUN (mg/dl) & 17.52 & \pm & 0.46 & 18.25 & \pm & 0.45 & 17.29 & \pm & 0.63 & 16.59 & \pm & 0.32 & 16.94 & \pm & 0.50 & 19.62 & \pm & $0.31^{*}$ \\
\hline CRE (mg/dl) & 0.57 & \pm & 0.03 & 0.56 & \pm & 0.02 & 0.54 & \pm & 0.02 & 6.09 & \pm & 5.55 & 0.53 & \pm & 0.01 & 0.61 & \pm & 0.02 \\
\hline $\mathrm{TP}(\mathrm{g} / \mathrm{dl})$ & 6.11 & \pm & 0.12 & 5.85 & \pm & 0.15 & 6.19 & \pm & 0.09 & 6.11 & \pm & 0.10 & 6.37 & \pm & 0.13 & 6.25 & \pm & 0.18 \\
\hline ALB (g/dl) & 3.07 & \pm & 0.05 & 3.03 & \pm & 0.06 & 3.06 & \pm & 0.03 & 3.07 & \pm & 0.05 & 3.10 & \pm & 0.04 & 3.21 & \pm & 0.06 \\
\hline T-BIL (mg/dl) & 0.20 & \pm & 0.03 & 0.18 & \pm & 0.02 & 0.18 & \pm & 0.02 & 0.14 & \pm & 0.02 & 0.16 & \pm & 0.02 & 0.18 & \pm & 0.02 \\
\hline D-BIL (mg/dl) & 0.09 & \pm & 0.01 & 0.09 & \pm & 0.00 & 0.10 & \pm & 0.01 & 0.09 & \pm & 0.00 & 0.08 & \pm & 0.01 & 0.18 & \pm & 0.07 \\
\hline AST $(U / L)$ & 193.00 & \pm & 13.81 & 158.60 & \pm & 9.13 & 152.30 & \pm & $13.61^{*}$ & 142.60 & \pm & $8.27^{*}$ & 146.80 & \pm & $10.34^{*}$ & 201.80 & \pm & 22.3 \\
\hline ALT (U/L) & 63.20 & \pm & 6.35 & 61.90 & \pm & 5.82 & 54.60 & \pm & 5.27 & 51.30 & \pm & 2.86 & 54.40 & \pm & 3.99 & 83.40 & \pm & $9.42^{*}$ \\
\hline ALP (U/L) & 55.80 & \pm & 2.51 & 53.20 & \pm & 2.28 & 52.90 & \pm & 1.59 & 55.10 & \pm & 2.61 & 61.10 & \pm & 2.22 & 64.90 & \pm & $3.40^{*}$ \\
\hline
\end{tabular}

A satellite group was given the $B$. monnieri extract at $1,500 \mathrm{mg} / \mathrm{kg}$ daily over 270 days followed by no treatment for 28 days

GLU glucose, BUN blood urea nitrogen, $C R E$ creatinine, $T P$ total protein, $A L B$ albumin, $T$-BIL total bilirubin, $D-B I L$ direct bilirubin, $A S T$ aspartate aminotransferase,

$A L T$ alanine aminotransferase, ALP alkaline phosphatase

Values are expressed as mean \pm S.E.M., $n=10$. *Significantly different from control, $p<0.05$

The internal organs were weighed and examined for gross pathology. Some test substances may harm tissues at the cellular level but not show any observable abnormalities. For this reason histopathological examination should be carried out to identify any cellular damage of the internal organs or tissues. In this study, internal organs including the brain, lungs, heart, liver, kidney, pancreas, spleen, stomach, duodenum, small intestine, and sex organs were examined. The examination found no significant histopathological changes in these organs or tissues. Although some statistically significant but minor differences in the body weight and the weight of some of the internal organs were observed, the values of those parameters were in normal range.

Hematological examination is one of the methods used to evaluate aspects of health status that may not be visible during physical examination [34]. This is important because the hematopoietic system is very sensitive to toxic substances. It can be affected by the ingestion of some toxic plants [35]. In particular, hematological parameters can provide important information about bone marrow activity as well as about intravascular effects such as hemolysis and anemia [36]. Morphological examination of RBC and WBC can identify their production or destruction. BASA, EOS, LYMP, MONO, and PMN are also used to assess the effect of test substances on immune systems [36-38]. Our results found significant differences in some hematological parameters, but values remained within normal limits [39].

Clinical blood chemistry examination was performed to quantify any toxic effects on pancreas function (GLU), kidney function (BUN, CRE) and liver function (SGOT, SGPT, ALP, TP, ALB, T-BIL and D-BIL). Plasma GLU is monitored to demonstrate the effect of a test substance on glucose metabolism. In terms of kidney function, the most common cause of acute or chronic renal failure is elevated BUN and increased CRE. Liver function is measured using a blood test which evaluates various functions of the liver, e.g., metabolism, detoxification, storage, and production of several vital protein components of blood plasma. Conditions commonly associated with testing for abnormalities of liver function 


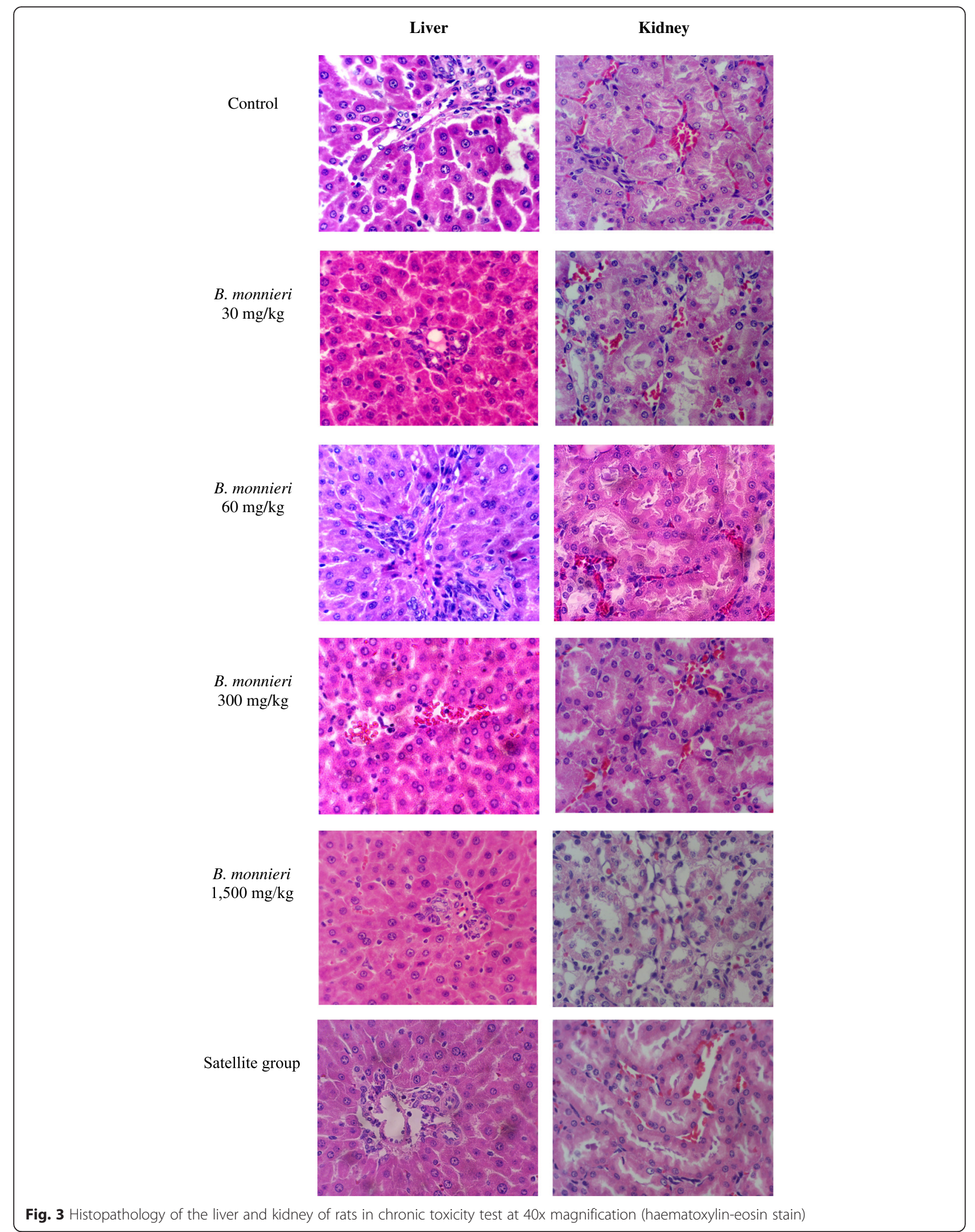


include gallbladder disease, hepatitis, fatty liver, cirrhosis, infectious mononucleosis and alcoholism $[38,40]$. In this study, significant differences in some clinical blood chemistry values were observed in both sexes between the experimental and the control groups. However, the alteration of these parameters was minor and remained within the normal range [41]. The data suggest that $B$. monnieri extract does not alter the function of the pancreas, liver or kidney.

The satellite-treated group was assessed for reversibility of the toxic effects of the test substance and for the occurrence of delayed toxic effects. In this study, the satellite-treated group received B. monnieri extract at a dose of $1,500 \mathrm{mg} / \mathrm{kg} / \mathrm{d}$ for 270 days. The rats were then kept for an additional 28 days post treatment. No toxic signs or symptoms or any abnormalities were detected.

Taken together, all of our results from both the acute and the chronic toxicity tests indicate that the $B$. monnieri extract is fairly non toxic for chronic use.

\section{Conclusion}

B. monnieri extract did not cause any signs of toxicity or any other symptoms in the acute and chronic oral toxicity tests of both female and male rats. That indicates that B. monnieri extract is relatively non-toxic. Further study regarding the toxicology of this extract should be conducted in non-rodent species or in humans.

\section{Abbreviations}

ALB, albumin; ALP, alkaline phosphatase; ALT, alanine aminotransferase; AST aspartate aminotransferase; BASO, basophil; BUN, blood urea nitrogen; CRE, creatinine; D-BIL, direct bilirubin; EOS, eosinophil; GLU, glucose; HB, hemoglobin $\mathrm{HCT}$, hematocrit; LYMP, lymphocyte; MCH, mean corpuscular hemoglobin $\mathrm{MCHC}$, mean corpuscular hemoglobin concentration; MCV, mean corpuscular volume; MONO, monocyte; PLT, platelet; PMN, neutrophil; RBC, Red blood cell count; T-BIL, total bilirubin; TP, total protein; WBC, white blood cell count
\end{abstract}

\section{Acknowledgements}

This study was supported by the National Research Council of Thailand (NRCT).

Funding

This study was granted from the National Research Council of Thailand (NRCT).

\section{Availability of data and materials}

Data are all contained within the paper.

\section{Authors' contributions}

All authors designed and coordinated all laboratory experiments. All authors conducted, analyzed, interpreted the results. All authors read and approved the final manuscript.

\section{Competing interests}

The authors declare that they have no competing interests.

\section{Consent for publication}

Not applicable.

\section{Ethics approval and consent to participate}

All experimental protocols on animals in this study were complied with the standards for the care and use of experimental animals and were approved by the Animal Ethics Committee of Faculty of Medicine, Thammasat University, Thailand (AE011/2552).

\section{Author details}

'Department of Pharmacology, Faculty of Medicine, Chiang Mai University, Chiang Mai 50200, Thailand. 'Division of Pharmacology, Department of Preclinical Science, Faculty of Medicine, Thammasat University, Pathum Thani 12120, Thailand. ${ }^{3}$ McCormick Faculty of Nursing, Payap University, Chiang Mai 50000, Thailand. ${ }^{4}$ Department of Pathology, Faculty of Medicine, Chiang Mai University, Chiang Mai 50200, Thailand. ${ }^{5}$ Bioscreening Unit, Department of Pharmaceutical Chemistry and Pharmacognosy, Faculty of Pharmaceutical Sciences and Centre of Excellence for Innovation in Chemistry, Naresuan University, Phitsanulok 65000, Thailand.

Received: 25 October 2015 Accepted: 21 July 2016

Published online: 27 July 2016

\section{References}

1. Sivarajan W. Balachandran I. Ayurvedic drugs and their plant sources. New Delhi: Oxford and IBH Publishing Co; 1994. p. 97.

2. Thomas RB, Joy S, Ajayan MS, Paulose CS. Neuroprotective Potential of Bacopa monnieri and Bacoside A against dopamine receptor dysfunction in the cerebral cortex of neonatal hypoglycaemic rats. Cell Mol Neurobiol. 2013;33(8):1065-74.

3. Saini N, Singh D, Sandhir R. Neuroprotective effects of Bacopa monnieri in experimental model of dementia. Neurochem Res. 2012;37(9):1928-37.

4. Le XT, Pham HT, Do PT, Fujiwara H, Tanaka K, Li F, Van Nguyen T, Nguyen KM, Matsumoto K. Bacopa monnieri ameliorates memory deficits in olfactory bulbectomized mice: possible involvement of glutamatergic and cholinergic systems. Neurochem Res. 2013;38(10):2201-15.

5. Piyabhan P, Wetchateng T. Cognitive enhancement effects of Bacopa monnieri (Brahmi) on novel object recognition and VGLUT1 density in the prefrontal cortex, striatum, and hippocampus of sub-chronic phencyclidine rat model of schizophrenia. J Med Assoc Thai. 2013;96(5):625-32.

6. Piyabhan $P$, Wetchateng $T$, Sireeratawong $S$. Cognitive enhancement effects of Bacopa monnieri Brahmi) on novel object recognition and NMDA receptor immunodensity in the prefrontal cortex and hippocampus of sub-chronic phencyclidine rat model of schizophrenia. J Med Assoc Thai. 2013;96(2):231-8.

7. Kamkaew N, Scholfield CN, Ingkaninan K, Taepavarapruk N, Chootip K. Bacopa monnieri increases cerebral blood flow in rat independent of blood pressure. Phytother Res. 2013;27(1):135-8.

8. Priyanka HP, Bala P, Ankisettipalle S, Thyaga Rajan S. Bacopa monnieri and L-deprenyl differentially enhance the activities of antioxidant enzymes and the expression of tyrosine hydroxylase and nerve growth factor via ERK 1/2 and NF-kB pathways in the spleen of female Wistar rats. Neurochem Res. 2012;38(1):141-52.

9. Jadiya P, Khan A, Sammi SR, Kaur S, Mir SS, Nazir A. Anti-parkinsonian effects of Bacopa monnieri: insights from transgenic and pharmacological Caenorhabditis elegans models of Parkinson's disease. Biochem Biophys Res Commun. 2011;413(4):605-10.

10. Kamkaew N, Scholfield CN, Ingkaninan K, Maneesai P, Parkington HC, Tare M, Chootip K. Bacopa monnieri and its constituents is hypotensive in anaesthetized rats and vasodilator in various artery types. J Ethnopharmacol. 2011;137(1):790-5

11. Menon BR, Rathi MA, Thirumoorthi L, Gopalakrishnan VK. Potential effect of Bacopa monnieri on nitrobenzene induced liver damage in rats. Indian J Clin Biochem. 2010;25(4):401-4.

12. Singh A, Singh SK. Evaluation of antifertility potential of Brahmi in male mouse. Contraception. 2009;79(1):71-9.

13. Sumathi T, Balakrishna K, Veluchamy G, Niranjali Devaraj S. Inhibitory effect of Bacopa monniera on morphine induced pharmacological effects in mice. Nat Prod Sci. 2007:13(1):46-53.

14. Russo A, Borrelli F, Campisi A, Acquaviva R, Raciti G, Vanella A. Nitric oxiderelated toxicity in cultured astrocytes: Effect of Bacopa monniera. Life Sci. 2003;73:1517-26.

15. Pawar R, Gopalakrishnan C, Bhutani KK. Dammarane triterpene saponin from Bacopa monniera as the superoxide Inhibitor in polymorphonuclear cells. Planta Med. 2001;67:752-4.

16. Sairam K, Dorababu M, Goel RK, Bhattacharya SK. Antidepressant activity of standardized extract of Bacopa monniera in experimental models of depression in rats. Phytomedicine. 2002;9:207-11.

17. Chowdhuri DK, Parmar D, Kakkar P, Shukla R, Seth PK, Srimal RC. Anti-stress effects of bacosides of Bacopa monnieri: modulation of HSP to expression, Sol and cytochrome P450 activity in rat brain. Phytother Res. 2002;16:639-45. 
18. Sairam K, Rao VC, Babu DM, Goel KR. Prophylactic and curative effects of Bacopa monniera in gastric ulcer models. Phytomedicine. 2001;8:423-30.

19. Elangovan V, Govindasamy S, Ramamoorthy N, Balasu-bramanian K. In vitro studies on the anticancer activity of Bacopa monniera. Fitoterapia. 1995;66:211-5.

20. Jain P, Khanna NK, Trehan T, Pendse VK, Godhwani JL. Anti-inflammatory effects of an Ayurvedic preparation, Brahmi Rasayan, in rodents. Indian J Exp Biol. 1994;32:633-6.

21. Roodenrys S, Booth D, Bulzomi S, Phipps A, Micallef C, Smoker J. Chronic Effects of Brahmi (Bacopa monnieri) on Human Memory. Neuropsychopharmacology. 2002;27:279-81.

22. Morgan A, Stevens J. Does Bacopa monnieri improve memory performance in older persons? Results of a randomized, placebo-controlled, double-blind trial. J Altern Complement Med. 2010;16(7):753-9.

23. Pase MP, Kean J, Sarris J, Neale C, Scholey AB, Stough C. The cognitiveenhancing effects of Bacopa monnieri: a systematic review of randomized, controlled human clinical trials. J Altern Complement Med. 2011;18(7):647-52.

24. Teschke R, Bahre R. Severe hepatotoxicity by Indian Ayurvedic herbal products: a structured causality assessment. Ann Hepatol. 2009;8(3):258-66.

25. Phrompittayarat W, Putalun W, Tanaka H, Wittaya-areekul S, Jetiyanon K, Ingkaninan K. An enzyme-linked immunosorbant assay using polyclonal antibodies against bacopaside I. Anal Chim Acta. 2007:584:1-6.

26. Phrompittayarat W, Putalun W, Tanaka $\mathrm{H}$, Wittaya-areekul S, Jetiyanon $\mathrm{K}$, Ingkaninan K. Determination of pseudojujubogenin glycosides from Brahmi based on immunoassay using a monoclonal antibody against bacopaside I. Phytochem Anal. 2007;18:411-8.

27. Organization of Economic Co-operation and Development. The OECD guideline for testing of chemical: 420 Acute Oral Toxicity-Fixed Dose Method. Paris France; 2001a. Available at http://www.oecd-library.org/ environment/test-no-420-acute-oral-toxicity-fixed-dose-procedure_ 9789264070943-en. Accessed 25 Nov 2015.

28. World Health Organization (WHO). General guidelines for methodologies on research and evaluation of traditional medicine. EDM/TRM/2000.1. Switzerland; 2000. Available at http://apps.who.int/medicinedocs/en/d/ Jwhozip42e/. Accessed 25 Nov 2015.

29. Organization of Economic Co-operation and Development. The OECD guideline for testing of chemical: 452 Chronic Toxicity Studies. France; 1981. Available at http://www.oecd-ilibrary.org/environment/test-no-452-chronictoxicity-studies 9789264071209-en. Accessed 25 Nov 2015.

30. Reagan-Shaw S, Nihal M, Ahmad N. Dose translation from animal to human studies revisited. FASEB. 2008;22:659-61

31. Tofovic SP, Jackson EK. Effects of long-term caffeine consumption on renal function in spontaneously hypertensive heart failure prone rats. J Cardiovascular Pharmacol. 1999;33:360-6.

32. Raza M, Al-Shabanah OA, El-Hadiyah TM, Al-Majed AA. Effect of prolonged vigabatrin treatment on hematological and biochemical parameters in plasma, liver and kidney of Swiss albino mice. Sci Pharm. 2002;70:135-45.

33. Teo S, Stirling D, Thomas S, Hoberman A, Kiorpes A, Khetani V. A 90-day oral gavage toxicity study of D-methylphenidate and D. L-methylphenidate in Sprague-Dawley rats. Toxicology. 2002;179:183-96.

34. Kronfeld DS, Medway W. Blood chemistry. In: Medway W, Prier JE, Wilkinson JS (12th eds.): Textbook of Veterinary Clinical Pathology. Baltimore: Williams \& Wilkins Co.; 1969

35. Adeneye AA, Ajagbonna OP, Adeleke TI, Bello SO. Preliminary toxicity and phytochemical studies of the stem bark aqueous extract of Musanga cecropioides in rats. J Ethnopharmacol. 2006;105(3):374-9.

36. Gregg LV. Anemias and polychythenias. In: Hematology techniques and concepts for veterinary technicians. U.S.A: lowa State University Press; 2000. p. 95-101.

37. Williams JW, Nelson DA, Morris MW. Examination of the blood. In: William JW, editor. Hematology international. 4th ed. New York: Mc Graw-Hell; 1990. p. 9-24.

38. Levine BS. Animal clinical pathology. In: Derelanko MJ, Hollinger MA, editors. CRC Handbook of Toxicology. 2nd ed. U.S.A: CRC Press; 2002. p. 742-68.

39. National laboratory animal center. Hematological data of NLAC-MU laboratory animals. Mahidol University. Thailand. http://www.nlac.mahidol.ac. th/acth/index.php/quality-control/health-report/27-quality-control\#Anchor3. Accessed 23 July 2016

40. Young LY, Holland EG. The clinical use of drugs: Interpretation of clinical laboratory tests. 6th ed. Vancouver: Lippincott Williams \& Wilkins; 1995. p. 4-20.

41. National laboratory animal center. Clinical chemistry of NLAC-MU laboratory animals. Mahidol University. Thailand. http://www.nlac.mahidol.ac.th/acth/ index.php/quality-control/health-report/27-quality-control\#Anchor3. Accessed 23 July 2016

\section{Submit your next manuscript to BioMed Central and we will help you at every step:}

- We accept pre-submission inquiries

- Our selector tool helps you to find the most relevant journal

- We provide round the clock customer support

- Convenient online submission

- Thorough peer review

- Inclusion in PubMed and all major indexing services

- Maximum visibility for your research

Submit your manuscript at www.biomedcentral.com/submit
(O) BioMed Central 